\title{
Tumour characterisation, staging and operability assessment in ovarian carcinoma: whole body diffusion-weighted $\mathrm{MRI}$ versus $\mathrm{CT}$
}

\author{
K Michielsen ${ }^{1 *}$, I Vergote ${ }^{2}$, R Vanslembrouck ${ }^{1}$ E E Mussen ${ }^{1}$, F Amant ${ }^{2}$, K Leunen ${ }^{2}$, P Moerman ${ }^{3}$, S Fieuws ${ }^{4}$, \\ F De Keyzer ${ }^{1}$, G Souverijns ${ }^{5}$, S Dymarkowski ${ }^{1}$, V Vandecaveye $^{1}$ \\ From International Cancer Imaging Society Meeting and 15th Annual Teaching Course (ICIS 2015) \\ London, UK. 5-7 October 2015
}

\section{Aim}

To prospectively evaluate whole body diffusion-weighted MRI (WB-DWI/MRI) for tumour characterisation, staging and prediction of complete (R0)-resection compared with computed tomography $(\mathrm{CT})$ in patients with suspected ovarian carcinoma.

\section{Methods}

One-hundred-sixty-six patients suspected for ovarian carcinoma underwent 3T WB-DWI/MRI using $2 \mathrm{~b}$-values $\left(b=0-1000 \mathrm{~s} / \mathrm{mm}^{2}\right), T 2$-weighted and contrast-enhanced T1-weighted sequences in addition to contrast-enhanced CT. WB-DWI/MRI and CT were independently and blindly evaluated and correlated with pathological findings at surgery as reference standard. Superiority was assessed using two-tailed McNemar tests for following parameters: characterisation of the malignant nature and primary origin of the ovarian mass, assessment of disease extent according to FIGO stage and prediction of R0-resection according to predefined operability criteria. Inter observer agreement for WB-DWI/MRI and CT was determined using Cohen's kappa statistics.

\section{Results}

For characterisation of malignancy, WB-DWI/MRI showed significantly higher accuracy compared with CT (93 versus $82 \%, p=0.001)$. WB-DWI/MRI correctly depicted a nonovarian malignant mass in $24 / 32(75 \%)$ of cases compared to only $6 / 32(19 \%)$ for CT $(\mathrm{p}<0.001)$. WB-DWI/MRI assigned more ovarian carcinoma patients to the correct
FIGO stage (71/94, 76\%) compared with CT (39/94, 41\%). For prediction of R0-resection, WB-DWI/MRI showed significantly higher sensitivity (95 versus $80 \%$ ), specificity (92 versus $74 \%$ ) and accuracy ( 94 versus $77 \%$ ) compared with CT ( $\mathrm{p}=0.039, \mathrm{p}=0.012$ and $\mathrm{p}<0.001$, respectively). Interobserver agreement was moderate to almost perfect $(\kappa=0.53-1.00)$ for WB-DWI/MRI and slight to moderate $(\kappa=0.04-0.52)$ for CT.

\section{Conclusion}

WB-DWI/MRI is superior to CT for lesion characterisation, staging and operability assessment of ovarian cancer justifying its development for pre-operative assessment of ovarian cancer patients.

\section{Authors' details}

'Department of Radiology, Leuven Cancer Institute, University Hospitals Leuven, KU Leuven, Belgium. 'Department of Obstetrics and Gynaecology, Division of Gynaecologic Oncology, Leuven Cancer Institute, University Hospitals Leuven, KU Leuven, Belgium. ${ }^{3}$ Department of Pathology, Leuven Cancer Institute, University Hospitals Leuven, KU Leuven, Belgium.

${ }^{4}$ Department of Public Health and Primary Care, Leuven Cancer Institute, University Hospitals Leuven, KU Leuven, Belgium. ${ }^{5}$ Department of Radiology, Jessa Hospitals, Hasselt, Belgium.

Published: 2 October 2015

doi:10.1186/1470-7330-15-S1-P40

Cite this article as: Michielsen et al:: Tumour characterisation, staging and operability assessment in ovarian carcinoma: whole body diffusionweighted MRI versus CT. Cancer Imaging 2015 15(Suppl 1):P40.

\footnotetext{
* Correspondence: katrijn.michielsen@med.kuleuven.be

'Department of Radiology, Leuven Cancer Institute, University Hospitals

Leuven, KU Leuven, Belgium

Full list of author information is available at the end of the article
} 